\title{
Population imbalance for a family of one-dimensional incommensurate models with mobility edges
}

\author{
Sayantan Roy, ${ }^{1,2,3,{ }^{*}}$ Subroto Mukerjee, ${ }^{2, \dagger}$ and Manas Kulkarni $\circledast^{3, \$}$ \\ ${ }^{1}$ Department of Physics, The Ohio State University, Columbus, Ohio 43210, USA \\ ${ }^{2}$ Department of Physics, Indian Institute of Science, Bangalore 560012, India \\ ${ }^{3}$ International Centre for Theoretical Sciences, Tata Institute of Fundamental Research, Bangalore 560089, India
}

(Received 25 November 2020; revised 2 April 2021; accepted 13 April 2021; published 10 May 2021)

\begin{abstract}
In this paper, we look at four generalizations of the one-dimensional Aubry-André-Harper (AAH) model which possess mobility edges. We map out a phase diagram in terms of population imbalance and look at the system size dependence of the steady-state imbalance. We find nonmonotonic behavior of imbalance with the system parameters, which contradicts the idea that the relaxation of an initial imbalance is fixed only by the ratio of the number of extended states to the number of localized states. We propose that there exist dimensionless parameters, which depend on the fraction of single-particle localized states, single-particle extended states, and the mean participation ratio of these states. These ingredients fully control the imbalance in the long time limit and we present numerical evidence of this claim. Among the four models considered, three of them have interesting duality relations and their locations of mobility edges are known. One of the models (next-nearest-neighbor hopping) has no known duality but a mobility edge exists and the model has been experimentally realized. Our findings are an important step forward to understanding nonequilibrium phenomena in a family of interesting models with incommensurate potentials.
\end{abstract}

DOI: 10.1103/PhysRevB.103.184203

\section{INTRODUCTION}

It is a well-known fact that for an arbitrarily small random disorder, the single-particle eigenstates of a noninteracting Hamiltonian are all localized in $d<3$ [1]. This phenomenon, termed Anderson localization, has been observed in a wide variety of systems $[2,3]$. For $d=3$, there is a critical value of disorder, above which a localization transition occurs, with a sharp energy-dependent mobility edge [4].

However, after the seminal work by Anderson, it was shown by Aubry and André that localization can occur even in the presence of a quasiperiodic potential [5]. Their model is described by the Hamiltonian

$$
H=\sum_{i}\left[t\left(c_{i+1}^{\dagger} c_{i}+\text { H.c. }\right)+\lambda \cos (2 \pi \beta i+\phi)\right],
$$

where particles are annihilated (created) by the operators $c_{i}$ $\left(c_{i}^{\dagger}\right)$ and they hop with amplitude $t$ on a one-dimensional (1D) lattice whose sites are labeled by the index $i . \lambda$ is the strength of the on-site potential and $\beta$ is an irrational number, which ensures the incommensurability of the potential with the lattice. This Hamiltonian also arises in the context of the quantum Hall effect on a lattice with an irrational flux per plaquette [6,7]. For the Aubry-André-Harper (AAH) model, there exists a critical disorder strength, below which all singleparticle eigenstates are delocalized, and above which all single-particle eigenstates are localized, which is in contrast

\footnotetext{
*roy.369@osu.edu

†smukerjee@iisc.ac.in

†manas.kulkarni@icts.res.in
}

to the phenomenon of Anderson localization in one dimension where all single-particle states are localized.

The AAH model possesses a duality that maps its localized and delocalized phases onto each other with the transition between them appearing at a self-dual point. The self-dual point occurs at $\lambda=2|t|$, where the eigenstates are neither localized nor delocalized but are critical [8], and transport is subdiffusive at the critical point [9]. The above type of quasiperiodic potential has recently been realized in optical lattices in the context of the experimental observation of many-body localization [10-12], dynamic potentials [13], with non-Abelian gauge potentials [14], and topologically protected edge states $[15,16]$. It was recently shown that the single-particle localization to delocalization transition follows a Kibble-Zurek mechanism for a quenched Aubry-André-like disordered potential [17]. Variants of this model which contain a single-particle mobility edge have also been realized experimentally [18-20].

The AAH model can be modified to produce single-particle mobility edges, and this has been studied recently in a wide context theoretically [21-37]. In fact, it is believed that a generic one-dimensional model with a quasiperiodic potential possesses single-particle mobility edges and their lack in the AAH model is due to its specific (fine-tuned) form [18,21,38].

It is paramount to mention that one of the major advantages of studying such models is that they mimic certain aspects of the behavior of generic disordered systems in three dimensions, such as the presence of a mobility edge while affording the computational advantage of being in one dimension.

It is well established that the properties of delocalization and localization are deeply connected to those of a measure of ergodicity and nonergodicity, respectively, in interacting 
systems [39-41]. Ergodicity ensures that at long times the system with any memory of its initial conditions settles into an equilibrium state. Generically, this equilibrium state does not possess broken translational order (note that the equilibrium state under discussion is a generic one at any energy rather than, say, the ground state, which is special and can have specific types of order). Therefore, one promising method to determine whether a given system is ergodic is to start it out in a state with broken translation order and observe whether that order disappears at long times. This is the method employed in several experimental and theoretical studies of many-body localization (MBL) via introducing the concept of imbalance as a diagnostic. For the lattice systems we consider here, the imbalance $I(t)$ at any time $t$ is a measure of this order and is defined as $I(t)=\frac{n_{e}(t)-n_{o}(t)}{L}$, where $n_{e}(t)$ and $n_{o}(t)$ are the total number of particles at even and odd sites, respectively, and $L$ is the size of the system.

The quantity imbalance is gaining importance for studying systems that break ergodicity, in the context of cold atom experiments, where such physics can be realized. A useful and straightforward indicator of localization of eigenstates is the mean participation ratio (inverse mean participation ratio), which is of $O(1)[O(L)]$ for localized states, and $O(L)[O(1)]$ for delocalized states. But unlike the imbalance, this quantity is not possible to measure in experiments. In our work, we will use the imbalance as a diagnostic tool to explore localization physics in one-dimensional quasiperiodic models that break ergodicity, as this quantity can be measured experimentally in a straightforward way [10], unlike the mean participation ratio or inverse participation ratio. Many of the results from our work can thus, in principle, be directly verified in experiments as well. The main aim of this paper is to establish the connection between ergodicity breaking, which can be seen in experiments by measuring the imbalance, and the degree of localization, of which the mean participation ratio is a measure.

For an ergodic system, an initially nonzero value of $I(t)$ is expected to go to zero at long times, while for a nonergodic one, it remains nonzero. Since nonergodicity is an essential feature of any localized system, this imbalance of a localized system, even one without interactions, is expected to remain nonzero. However, the situation for noninteracting systems with delocalized states (regardless of whether they occupy the entire energy spectrum or only a part of it) is less obvious since these systems are not strictly ergodic (not possessing interactions). We investigate the behavior of the imbalance for different types of localized, delocalized, and "mixed" systems in this work. Although the new order parameter, imbalance, was studied earlier in the context of interacting systems, its relevance to noninteracting models $(U=0)$ was already shown in Ref. [10]. Systems with single-particle mobility edges for a one-dimensional quasiperiodic potential [18] were also studied once the importance of imbalance for studying localization physics in optical lattices was established. A detailed analysis of noninteracting models, having singleparticle mobility edges, may not have been important before, but with such models [20] synthesized now, we expect our results to motivate experiments studying imbalance in them as well.

Although we do not study interacting models in this work, we would like to point out that the physics observed in non- interacting models might be relevant for models where an interparticle interaction is present. The many-body states of noninteracting models, with single-particle localized states, are many-body localized themselves, and show properties such as Poissonian energy level statistics, violation of the eigenstate thermalization hypothesis (ETH), area law scaling of entanglement, etc., which are signatures of MBL states. Thus, understanding any experimental diagnostics of many-body states of a noninteracting model, in which only single-particle localized states are occupied (these are MBL states themselves), might explain observations and help in understanding phase transitions in MBL experiments.

In this paper, we calculate the steady-state imbalance for various noninteracting models with quasiperiodic potentials, obtained by modifying the AAH model. An initial state is chosen with a broken translational order, in which every even site is occupied by a particle, and the odd sites are empty. This charge density wave (CDW) state is similar to the one employed in Ref. [10]. The relaxation of the CDW can be calculated in the unitary time evolution that follows. The calculations are performed by numerically solving the Schrödinger equation on finite-sized systems and the imbalance is obtained as a function of the various microscopic parameters and the length $L$ of the system. We perform finite-size scaling to determine the value of the imbalance in the thermodynamic limit and its dependence on system size. Further, we examine its variation with the microscopic parameters of the models under consideration, and we find a nonmonotonic dependence on them. We show that the value of the imbalance is not simply determined by the relative fraction of localized to delocalized states but it also depends on the strength of localization and delocalization of these states. This is quantified by the measures $\epsilon$ and $\epsilon^{\prime}$ (to be defined later) whose values we show are correlated with that of the imbalance. In other words, in addition to how many localized or delocalized states exist, we demonstrate that it is also of paramount importance to quantify how "localized" a localized state is and how "delocalized" a delocalized state is.

The rest of the paper is organized as follows. In Sec. II we introduce the models (see also Table I), and point out their main features. We present a brief derivation of the exact mobility edge for the first two generalized AAH models, following Ref. [25], and for the slowly varying deterministic potential model from Ref. [42]. Section III is dedicated to describing the methods employed and calculations.

In Sec. IV, we calculate the fraction of localized states, whenever the exact expression for the mobility edge is known, and use it to construct the phase diagram for the model. In case the location of the mobility edge is not known, we use the mean participation ratio (which is introduced in Sec. II, model 3 ) to map out an approximate phase diagram. We also look at the evolution of a broken translation order in terms of a charge density wave (CDW) and calculate the imbalance in the long time limit. The long time value of imbalance serves as an order parameter and can be used to construct an approximate phase diagram, akin to the fraction of localized states. For the main part of this paper, we focus on calculations of model 1 only and show that all the justifications for the behavior of model 1 in different phases using this order parameter may be extended to the other three models in the Appendixes. 
TABLE I. Models studied and their properties. All the models feature delocalized, intermediate phases with a mobility edge, and localized phases, determined by the parameters $\lambda, \alpha$ [Figs. 1(a)-4(a)].

\begin{tabular}{llll}
\hline \hline Model & \multicolumn{1}{c}{ Hopping term } & On-site potential $V_{i}$ & Location of mobility edge \\
\hline 1 & $T_{i, i+1}=t\left(a_{i}^{\dagger} a_{i+1}+\right.$ H.c. $)$ & $2 \lambda \frac{\cos (2 \pi \beta i+\phi)}{1-\alpha \cos (2 \pi \beta i+\phi)} a_{i}^{\dagger} a_{i}$ & $\alpha E_{c}=2 \operatorname{sgn}(\lambda)(|t|-|\lambda|)$ \\
2 & $T_{i, i+1}=t\left(a_{i}^{\dagger} a_{i+1}+\right.$ H.c. $)$ & $2 \lambda \frac{(1-\cos (2 \pi \beta i+\phi)}{1+\alpha \cos (2 \pi \beta i+\phi)} a_{i}^{\dagger} a_{i}$ & $\alpha E_{c}=2 \operatorname{sgn}(\lambda)(|t|-|\lambda|)$ \\
3 & $T_{i, i+1}=t\left(a_{i}^{\dagger} a_{i+1}+\right.$ H.c. $)$ & $2 \lambda \cos (2 \pi \beta i+\phi) a_{i}^{\dagger} a_{i}$ & No analytical expression, \\
& $T_{i, i+2}=\mu t\left(a_{i}^{\dagger} a_{i+2}+\right.$ H.c. $)$ & & phases calculated through PR \\
4 & $T_{i, i+1}=t\left(a_{i}^{\dagger} a_{i+1}+\right.$ H.c. $)$ & $2 \lambda \cos \left(2 \pi \beta i^{\alpha}+\phi\right) a_{i}^{\dagger} a_{i}$ & $E_{c}= \pm 2(|t|-|\lambda|)$ \\
\hline \hline
\end{tabular}

We look at finite-size effects and propose a leading order scaling of the imbalance with system size. This is used to extract the thermodynamic limit of the imbalance $I_{0}$ which unveils a nontrivial behavior across the parameter space, noted first in model 1 in Ref. [43]. We extend this analysis for the other three models as well and show that the nonmonotonicity is apparent in them as well.

Also in Sec. IV, we provide an explanation for this counterintuitive behavior by using two dimensionless parameters, $\epsilon$ and $\epsilon^{\prime}$, following a recent application of them to distinguish between the ergodic and MBL phase [44]. These parameters capture the effective strength of the single-particle localized states versus extended states in confining the particles' motion, and hence are directly linked to the behavior of imbalance as a function of microscopic parameters of the model. In Sec. V, we summarize our results along with a brief outlook.

A discussion of the other three models is presented in the Appendixes, with Appendix A discussing the system size dependence of the steady-state imbalance and its behavior in different phases. Appendix B shows how the appearance of multiple mobility edges show a unique transition between phases in model 3.

\section{MODELS}

The models are chosen from Sec. II of Ref. [44]. We would like to point out that the choice of models is based primarily on the relevance of the systems to present day experiments, and how well they have been explored. For example, the specific choice of models in our work is motivated by the fact that the location of the single-particle mobility edge in them is known precisely. In practice, the set of models that have single-particle mobility edges is very large; almost any quasiperiodic model with a short-range hopping has a single-particle mobility edge [30,31,42,45]. The fine-tuned energy-independent duality of the Aubry-André model prevents the occurrence of a mobility edge but generically models with quasiperiodic potentials are expected to possess mobility edges. We thus limit ourselves to the study of only four well-characterized models, and given that they are not trivially related to one another, believe that our conclusion relating the imbalance to the degree of localization applies much more broadly for models with mobility edges. All the models we study contain an on-site potential $V_{i}$ of strength $\lambda$. The form of the potential also contains an irrational number $\beta$ (which we set equal to the golden mean $\frac{\sqrt{5}+1}{2}$ ) that ensures its incommensurability with the underlying lattice and a phase $\phi$ which can be used to move the potential relative to the lattice. $\phi$ is averaged over in our calculation analogous to disorder averaging. Finally, the potential also depends on an auxiliary parameter $\alpha$, which can be tuned to move the position of the mobility edge in the spectrum. The models studied are listed in Table I along with some of their specific properties. We restrict to $\lambda \in[0,2|t|]$ and $\alpha \in(0,1]$ unless otherwise specified.

The first two of the four models we study were explored by Ref. [25]. The models were shown to be self-dual by a generalized transformation akin to a Fourier transformation, and unlike the AAH model, have mobility edges due to energy-dependent self-dual conditions. The first of this family of self-dual models is the generalized AAH model (GAAH), described by

$$
H^{(1)}=t \sum_{i}\left(a_{i}^{\dagger} a_{i+1}+\text { H.c. }\right)+\sum_{i} \frac{2 \lambda \cos (2 \pi \beta i+\phi)}{1-\alpha \cos (2 \pi \beta i+\phi)} a_{i}^{\dagger} a_{i}
$$

Another modification to this model, which preserves the self-duality condition, and transforms under the same transformation [25], is

$$
\begin{aligned}
H^{(2)}= & t \sum_{i}\left(a_{i}^{\dagger} a_{i+1}+\text { H.c. }\right)+2 \lambda \\
& \times \sum_{i} \frac{1-\cos (2 \pi \beta i+\phi)}{1+\alpha \cos (2 \pi \beta i+\phi)} a_{i}^{\dagger} a_{i} .
\end{aligned}
$$

The Schrödinger equation for both the models is written out, following Ref. [25] ( $g$ below contains the details of the specific model),

$$
t\left(\psi_{i-1}+\psi_{i+1}\right)+g \chi_{i}(\delta) \psi_{i}=(E+2 \lambda \cosh \delta) \psi_{i},
$$

with the definition of the on-site potential $\chi_{i}[25]$,

$$
\chi_{i}(\delta)=\frac{\sinh (\delta)}{\cosh (\delta)-\cos (2 \pi \beta i+\phi)},
$$

where $\delta$ is defined in Eq. (9). The location of the mobility edge can be found using the self-dual transformation of the amplitude of the wave function at site $i, \psi_{i}$, to that at point $k$ in the dual space [25],

$$
f_{k}=\sum_{m n i} e^{i 2 \pi b(k m+m n+n i)} \chi_{n}^{-1}\left(\delta_{0}\right) \psi_{i}
$$

which transforms the Schrödinger equation [Eq. (4)] to

$$
t\left(f_{k-1}+f_{k+1}\right)+g \frac{\sinh \delta}{\sinh \delta_{0}} \chi_{k}\left(\delta_{0} f_{k}\right)=2 t \cosh \delta f_{k},
$$


with the parameter $\delta_{0}$ defined as

$$
\begin{gathered}
\delta_{0}=\cosh ^{-1}\left(\frac{E+2 \lambda \cosh \delta}{2 t}\right), \\
\delta=\cosh ^{-1}(1 / \alpha) .
\end{gathered}
$$

The parameter $g$ is model dependent, and for the model in Eq. (2) is defined as

$$
\begin{aligned}
& g=2 \lambda \cosh (\delta) / \tanh (\delta) \quad(\text { model } 1), \\
& g=2 \lambda(1+\cosh \delta) / \sinh (\delta) \quad(\text { model } 2),
\end{aligned}
$$

for the model in Eq. (3). The self-dual condition of Eqs. (4)(7) is met by $\delta=\delta_{0}$, which gives the equation for the position of the mobility edge:

$$
\alpha E=2 \operatorname{sgn}(\lambda)(|t|-|\lambda|) \quad(\text { models } 1 \text { and 2). }
$$

For both models, states with energy $E>E_{c}$ are extended, and those with $E<E_{c}$ are localized. Both of these models are a subset of class of models with the on-site term as in Eq. (4) which may be obtained with an arbitrary choice of the parameters $g(\alpha, t)$ and $E(\lambda, \alpha, t)$ but still have an exact mobility edge. The parameter $\alpha$ is a generalization of various special cases: For model 1 [Eq. (2)], $\alpha=0$ produces the AAH limit. For model 2 [Eq. (3)], $\alpha=0$ corresponds to the AAH model, $\alpha=-1$ produces the constant on-site potential model with $V_{i}=2 \lambda$, and $\alpha=1$ produces the closed form singular potential [25] $V_{i}=\tan ^{2}\left(\frac{2 \pi \beta i+\phi}{2}\right)$.

Another perturbation to the self-duality of the AAH model may be introduced by considering long-range hopping, as studied in Ref. [46]. The Schrödinger equation becomes

$$
\sum_{i \neq j} t e^{-p|i-j|} \psi_{j}+2 \lambda \cos (2 \pi \beta i+\phi) \psi_{i}=E \psi_{i},
$$

where $0<p<1$. The inclusion of an exponentially decaying hopping amplitude perturbs the AAH duality to the following linear self-dual relation,

$$
\cosh (p)=\frac{E+t}{2 \lambda} .
$$

In our work we consider only a next-nearest-neighbor hopping term as the perturbation to the AAH model, also called as the $t_{1}-t_{2}$ model. Without loss of generality, we parametrize the hopping amplitudes as $t_{1}=t, t_{2}=\mu t$, with $\mu=e^{-p}$, and $1 / p$ is defined as the length scale over which the hopping strength decays in Eq. (11). In this parametrization, consider

$$
\begin{aligned}
H^{(3)}= & t \sum_{i}\left(a_{i}^{\dagger} a_{i+1}+\mu a_{i}^{\dagger} a_{i+2}+\text { H.c. }\right) \\
& +\sum_{i} 2 \lambda \cos (2 \pi \beta i+\phi) a_{i}^{\dagger} a_{i} .
\end{aligned}
$$

This model produces the nearest-neighbor hopping AAH model with $\mu=0$, and two superimposed AAH lattices with nearest- and next-nearest-neighbor hopping at $\mu=1$. This model does not have an exact expression for the mobility edge, although studies have shown the presence of both localized and delocalized states coexisting at the same value of the microscopic parameters $[30,46]$.

A first-order approximation to the hopping amplitude in Eq. (11) may be made by considering all hoppings longer than the next-nearest-neighbor interaction to be suppressed, by a large value of $p$. An approximate relation for the location of the mobility edge is constructed by substituting $p=\ln \left(t_{1} / t_{2}\right)$ into Eq. (12), which yields

$$
E_{c} \sim \lambda\left(\mu+\frac{1}{\mu}\right)-t \quad \text { (approximate for model 3). }
$$

As found in Ref. [46], this works only for small values of $\mu$, or large values of $p$, as shown by inverse participation ratio calculations. To explore the phases in this model, an exact diagonalization of the Hamiltonian is employed to get the eigenstates and calculate the participation ratio (PR) of the $n$th eigenstate, defined as

$$
\operatorname{PR}\left(\psi_{n}\right)=\frac{1}{\sum_{j}\left|\psi_{n}(j)\right|^{4}},
$$

where $\psi_{n}(j)$ is the amplitude of the $n$th eigenstate at site $j$. This is of $O(1)$ for the localized states, and $O(L)$ for delocalized states, with $L$ being the system size. Owing to the lack of an expression for the mobility edge for model 3 , the mean participation ratio (MPR), which is the PR averaged over all eigenstates $n, \mathrm{MPR}=\langle\mathrm{PR}\rangle_{\{n\}}$, will be used to map out a phase diagram for this model, instead of using the fraction of localized states, as done for the other models.

The next model we study contains a slowly varying deterministic potential in the on-site term. This class of models was studied in Ref. [42] and its geometrical properties without referring to tight-binding Hamiltonians were explained in Ref. [47]. The Hamiltonian is

$$
H^{(4)}=t \sum_{i}\left(a_{i}^{\dagger} a_{i+1}+\text { H.c. }\right)+\sum_{i} 2 \lambda \cos \left(2 \pi \beta i^{\alpha}+\phi\right) a_{i}^{\dagger} a_{i} \text {. }
$$

The model transitions smoothly from the constant uniform potential at $\alpha=0$ to the AAH limit at $\alpha=1$. For $\alpha>2$, the potential is "pseudorandom" in the sense that the localization length for the $\alpha>2$ case was shown to be the same as that in the corresponding random case [42], and this model can be identified with the 1D Anderson model [42]. For $1<\alpha<2$, it was proved that the eigenstates at the center of the spectra are all localized, but with a large localization length, or vanishing Lyapunov exponents [48]. The localization mechanism is different from the Anderson mechanism, as the potential is neither aperiodic, nor random, but is deterministic. Indeed, it was shown that the density of states has a discontinuity at the mobility edges, unlike the 3D Anderson model [42]. The slowly varying nature of the potential is written as

$$
\frac{d V_{i}}{d i}=\frac{-4 \lambda \pi \beta \cos \left(2 \pi \beta i^{\alpha}+\phi\right)}{i^{1-\alpha}},
$$

which implies that $\left(V_{i+1}-V_{i}\right) \propto i^{\alpha-1}$, and this in the limit of large system sizes, goes to zero, as $0<\alpha<1$. Note that this almost constant feature of the potential is crucial for the localization transition in this model. The Schrödinger equation is solved with the ansatz $\psi_{n}=z^{n}$, and it may be proved that it is possible to have localized states (extended states) for real $z$ 

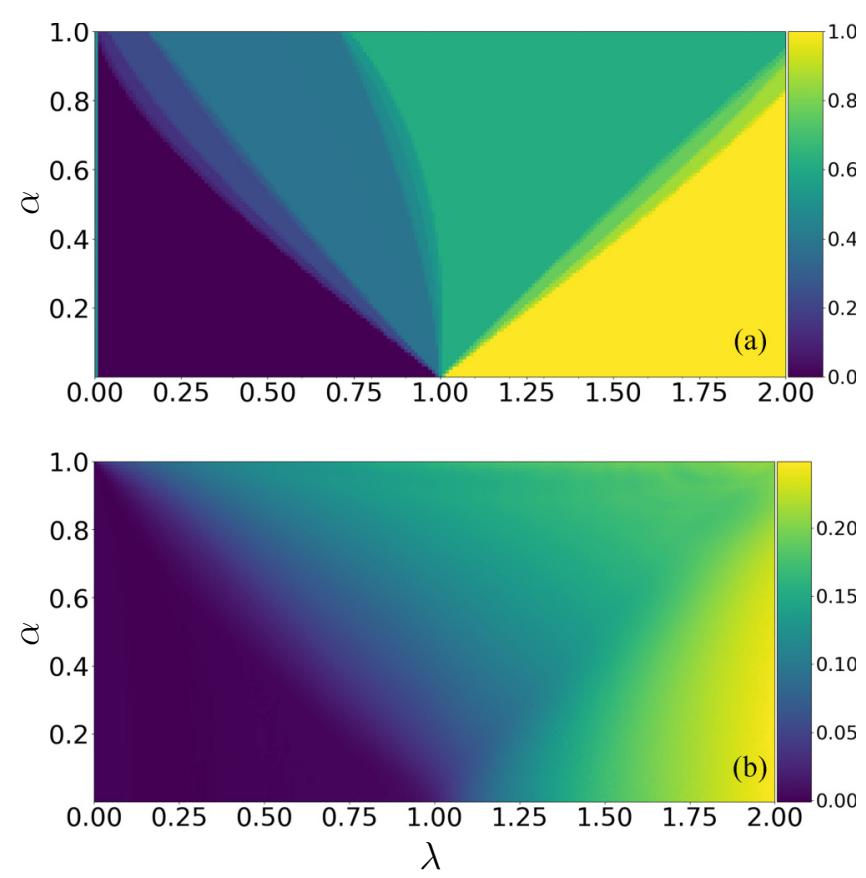

FIG. 1. (a) Phase diagram of model 1 for $\beta=\frac{\sqrt{5}+1}{2}$ as a function of $\lambda$ and $\alpha$ in terms of the fraction of localized states $\eta$ (single disorder realization). (b) Steady-state imbalance for model 1 ( $L=256$, averaged over 1000 disorder configurations).

(imaginary $z$ ) only at

$$
\begin{aligned}
& \operatorname{Re}(z)=0 \Rightarrow|E|>2(|t|-|\lambda|), \\
& \operatorname{Im}(z)=0 \Rightarrow|E|<2(|t|-|\lambda|),
\end{aligned}
$$

which gives us the location of the mobility edge [42] (independent of $\alpha$ ) at

$$
E_{c}= \pm 2(|t|-|\lambda|), \quad \text { for model } 4 .
$$

All states at the center of the spectra, with energy $E,|E|<$ $\left|E_{c}\right|$ are extended, and those at the tails with $|E|>\left|E_{c}\right|$ are localized [for the positive $(\lambda, \alpha)$ quadrant].

\section{METHODS AND CALCULATIONS}

We employ an exact diagonalization of the Hamiltonians in Eq. (2) (model 1), Eq. (3) (model 2), Eq. (13) (model 3), and Eq. (16) (model 4), with the parameters limited to $t=-1$, $\mu \in(0,1), \lambda \in[0,2|t|], \alpha \in(0,1]$. We perform calculations with lattice sizes up to $L=900$ and use a disorder averaging over $\phi$ to restore translation invariance. The number of disorder samples used for Figs. 1-4 is 1000, as we use a relatively small system size (256) for these plots. To study the scaling of the steady-state imbalance with the number of sites, the data are obtained over a set of larger system sizes. However, this limits the number of disorder samples for which the results can be averaged over in a fixed time, limiting the number of samples to 100. Increasing the sample size further does not appear to change the results in any discernible way. The error bars are not shown in the plots, because they are of the same size as that of the plot markers themselves, and hence we neglect them. All the results shown below are for $\beta=\frac{\sqrt{5}+1}{2}$ (golden mean) unless mentioned explicitly to be a different number. The imbalance is calculated by looking at the number density at each site, from the single-particle wave-function amplitude $\left|\psi_{n}(i)\right|^{2}$. The imbalance is obtained by summing over the amplitudes, over an entire single-particle spectrum. The steady-state value of the imbalance is calculated from the average in the time window $200 \tau-400 \tau$, where $\tau=\hbar /|t|$ is the unit of time. The imbalance is constant in this interval with only small fluctuations.

The system sizes that we study may be small in the context of the study of noninteracting problems, but we would like to point out that for all our results, we do not need very large system sizes. Our main goal of doing a finite-size scaling is to show that the steady-state imbalance follows a $1 / L$ scaling in the leading order, and hence including larger system sizes causes the data points in $I$ vs $1 / L$ scaling to lie closer to each other as $L$ is increased, and this does not change the overall scaling form at all. The single-particle wave functions can also be grouped into an extended/localized category by looking at their energy and the respective mobility edge expressions for the models, where it is known analytically. We also verify the distribution by looking at the PR calculation (not presented here), defined in Eq. (15). For model 3, where the exact mobility edge is not known, the PR is calculated to differentiate between the single-particle states, where it scales as

$$
\mathrm{PR} \sim \begin{cases}O(L), & \text { delocalized } \\ O(1), & \text { localized, } \\ O\left(L^{D(q)}\right), & \text { critical }\end{cases}
$$

where $D(q)$ is the fractal exponent. The phase diagram is then constructed by looking at the fraction of localized states $\eta$ (models 1, 2, and 4) and MPR (model 3) for all possible $(\lambda, \alpha)$. We also plot the steady-state imbalance over the parameter space for all models and see that it maps out an approximate phase diagram similar to the one obtained by using the diagnostic $\eta$.

After a finite-size scaling for the steady-state imbalance, the imbalance follows a linear scaling in $1 / L$ at leading order, whenever the steady-state value is high enough to differentiate it from statistical noise. We propose that this is the behavior at leading order at least in the mobility edge and localized edge phase. At the critical point, we numerically find that it shows $\frac{1}{L}$ even for relatively small system sizes. (This dependence on system size is a numerical observation for which we do not currently have an analytical understanding.) This allows us to write the steady-state imbalance as

$$
I(L)=\frac{a}{L}+I_{0}+\text { higher-order terms. }
$$

The coefficient $a$ quantifies the correlation of the steady-state imbalance with system size $L$, and is not relevant for our work. We will study the behavior of $I_{0}$, the thermodynamic limit of the steady-state imbalance. This behavior captures the trend seen in the imbalance phase diagram, for finite sizes. The behavior of $I_{0}$ is explained by calculating the two dimensionless 
parameters $\epsilon$ and $\epsilon^{\prime}$ as

$$
\begin{gathered}
\epsilon=\tilde{\eta} \frac{1-\mathrm{MPR}_{D} / L}{\mathrm{MPR}_{L}-1} \quad \text { (degree of delocalization) } \\
\epsilon^{\prime}=\frac{1}{\mathrm{MPR}_{L}-1} \quad \text { (degree of localization). }
\end{gathered}
$$

The parameter $\epsilon$ was used by Ref. [44] to define criteria for the MBL to ergodic phase transition in interacting systems, with a single-particle mobility edge. For our work, the parameters will link the imbalance observed in these models to the mean participation ratio of their single-particle spectrum, and hence characterize the degree of localization in these models as a function of the model parameters. The parameter $\epsilon$ satisfies the following,

$$
\begin{gathered}
\epsilon<1, \quad \text { ergodic phase, } \\
\epsilon>1, \quad \text { MBL phase. }
\end{gathered}
$$

Here, $\tilde{\eta}$ is the ratio of the number of localized to delocalized single-particle states, and $\mathrm{MPR}_{D}\left(\mathrm{MPR}_{L}\right)$ is the mean participation ratio averaged over the spectrum and $\phi$ disorders following Eq. (15) over the delocalized (localized) states only. Note that $\epsilon$ captures how strongly localized the single-particle localized states are versus how strongly delocalized the singleparticle delocalized states are, only in the mobility edge phase [44]. This is by construction $0(\tilde{\eta}=0)$ in the delocalized phase, and $\infty$ [due to Eq. (23)] in the localized phase.

To look at the degree of localization of the localized states only, a new parameter $\epsilon^{\prime}$ is defined in Eq. (24). We show that the parameters $\epsilon$ and $\epsilon^{\prime}$ are capable of explaining the behavior of $I_{0}$ for all the models in their various phases. The parameter $\epsilon$ has a jump discontinuity unlike $\epsilon^{\prime}$, whenever the fraction of localized states $\eta$, and hence $\tilde{\eta}$, change over the phase diagram in the mobility edge phase for all the models.

\section{RESULTS}

\section{A. Phase diagram}

An exact diagonalization, as described in the last section, is used to map out the phase diagrams for all the models, listed in Sec. II and Table I. These are presented in Figs. 1(a)-4(a). For models 1, 2, and 4, the phase diagram is represented in terms of the fraction of localized states $\eta$. We calculate it from Eq. (10) for models 1 and 2 and Eq. (20) for model 4, and in terms of the MPR, obtained from Eq. (15) for model 3 . The phase diagram of model 1 with $\beta=(\sqrt{5}-1) / 2$ has been obtained previously [44] and the phase diagram with $\beta=(\sqrt{5}+1) / 2$ has been obtained in Ref. [43]. The latter agrees with the one we have obtained and shown in Fig. 1(a). For model 3 [Fig. 3(a)], the phase diagram reveals a rich transition physics in the region of $\lambda<1$, where the system transitions from a delocalized to mobility edge to back to a delocalized phase. This is attributed to the appearance and merger of the mobility edge. The system departs from the delocalized phase to the mobility edge phase, as $\mu$ increases from 0 , due to the appearance of a single mobility edge.

At higher values of $\mu$, multiple mobility edges appear in the system, and they merge to bring the system back into the delocalized phase at some higher $\mu$. This is shown using the
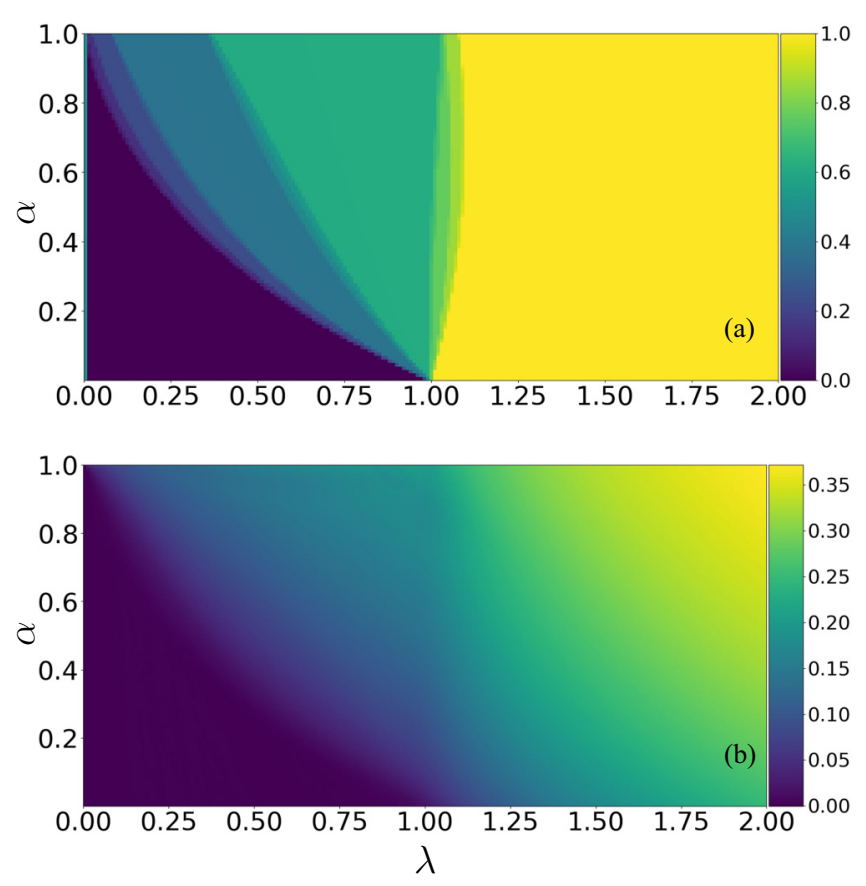

FIG. 2. (a) Phase diagram of model 2 for $\beta=\frac{\sqrt{5}+1}{2}$ as a function of $\lambda$ and $\alpha$ in terms of the fraction of localized states $\eta$ (single disorder realization). (b) Steady-state imbalance for model 2 ( $L=256$ sites, averaged over 1000 disorder configurations).

PR calculation, in Fig. 10 (see the Appendixes) for multiple values of $\mu$ along a fixed $\lambda$ at which this peculiar transition occurs.

Model 4 [Fig. 4(a)] shows an exclusively localized phase for $\lambda>1$, for both the choices of $\beta$.

However, it is also worth noting that the phase diagram is identical for both values of $\beta$, which implies that the spectrum is insensitive to the choice of irrational number. This is in agreement with the claim that the parameter $\beta$ is an irrelevant parameter for the model, from a scaling theory perspective [42].

\section{B. Imbalance}

The imbalance can also be used to identify the different phases. The imbalance thus maps out an approximate version of the phase diagram. At long times, it remains close to the initial value of 0.5 deep in the localized phase, asymptotes to 0 deep in the delocalized phase, and to intermediate values in the vicinity of the mobility edge. This is shown for all the models in Figs. 1(b)-4(b). Results of the previous discussion (which is the top panel of Figs. 1-4) show that the imbalance can be used to obtain a rough phase diagram with smeared boundaries. The direct juxtaposition of panels (a) and (b) of Figs. 1-4 makes it clear that the fraction of localized states fails to capture the complex behavior of the amount of localization in these systems (which is captured by the steady-state imbalance). There is thus no one-to-one correlation between the fraction of localized states and the imbalance, and the calculation of these phase diagrams show this difference.

We observe, as in Ref. [43], a nonmonotonic decrease in the imbalance even if we cross from a localized phase to a 

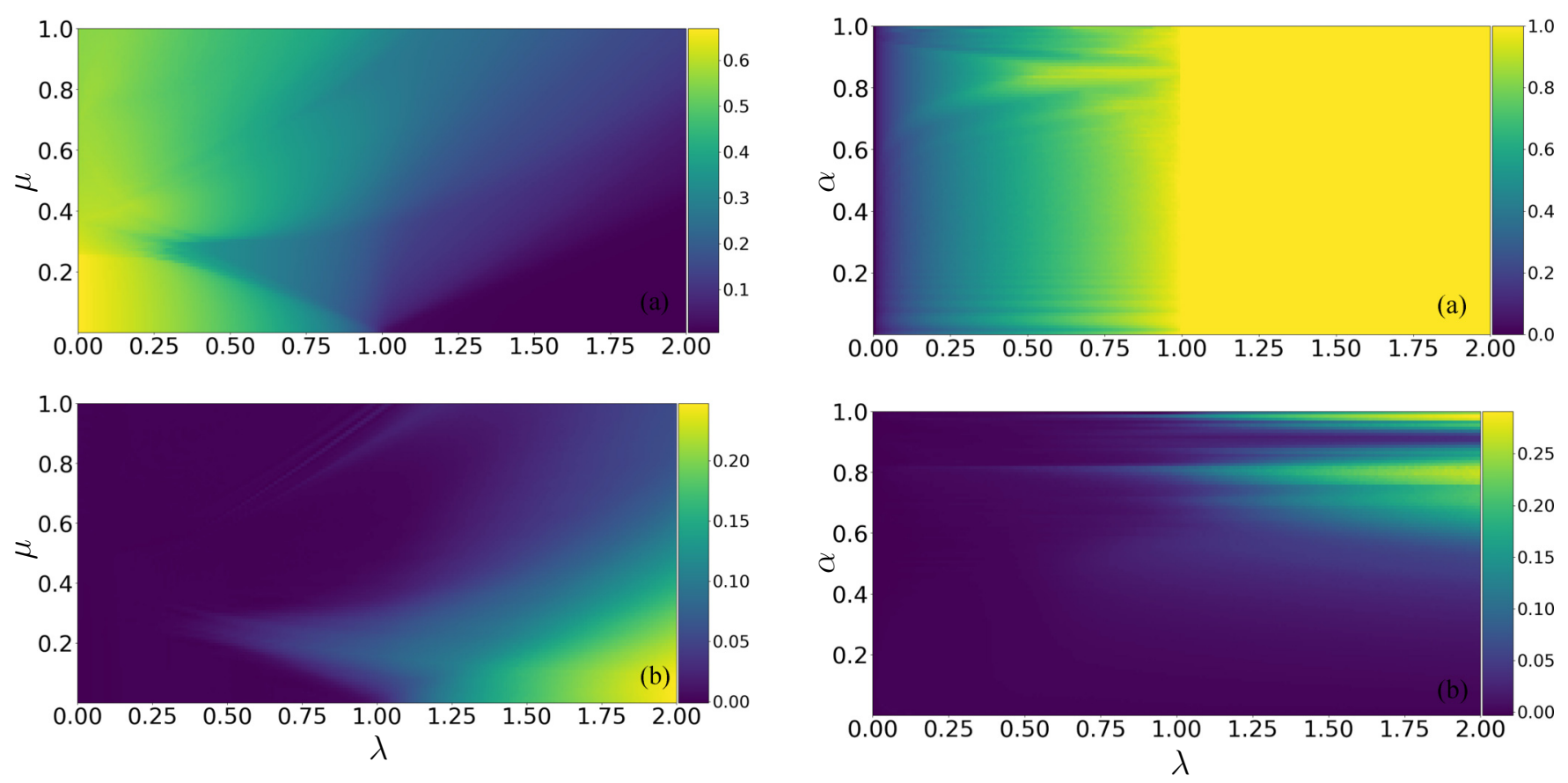

FIG. 3. (a) Phase diagram of model 3 for $\beta=\frac{\sqrt{5}+1}{2}$ as a function of $\lambda$ and $\mu$ in terms of MPR $/ L$. MPR $/ L \sim 0$ means a localized phase, and $\mathrm{MPR} / L \sim O(1)$ means a delocalized phase. (b) Steady-state imbalance for model 3 ( $L=256$ sites, averaged over 1000 disorder configurations).

mobility edge (ME) phase (one would expect a strict decrease, as the number of localized states decreases). The fact that the steady-state imbalance is not a trivial function of the fraction of localized states is also seen in models 2 and 4 . A uniform fraction of localized states should have implied a uniform value for the steady-state imbalance, but this not the case in the localized phase of models 2 and 4, as seen in Figs. 2(b) and 4(b).

Our main aim is to demonstrate a correlation between this nontrivial behavior and specific quantities obtained from the single-particle spectrum related to the degree of localization in the system. This is done by the two parameters $\epsilon$ and $\epsilon^{\prime}$, which link the steady-state imbalance to the degree of localization of the system as a function of parameters in the model.

To exclude any finite-size effects that may cause this nonmonotonic behavior of the steady-state imbalance, we obtain a scaling of the steady-state imbalance with system size. To leading order, the correction appears to be of the form $I=\frac{a}{L}+I_{0}$, presented in Fig. 5(a). Thus, the thermodynamic limit is the intercept of the straight line $I_{0}$. At the critical point, our numerics show that $1 / L$ behavior survives even for small system sizes, verified for models 1,2 , and 4 . Here, the localization length is of the order of system size along the line separating the localized and ME phase. The $1 / L$ scaling also seems to hold at small system sizes at the AAH critical point. The leading order correction seems to hold quite well whenever the imbalance has a large value, even in the localized and ME phases. We emphasize this is only the leading order term, and an analytical expression for this quantity in terms of the parameters of the model is currently lacking.

FIG. 4. (a) Phase diagram of model 4 for $\beta=\frac{\sqrt{5}+1}{2}$ as a function of $\lambda$ and $\alpha$ in terms of the fraction of localized states $\eta$ (single disorder realization). (b) Steady-state imbalance for model 4 ( $L=256$ sites, averaged over 1000 disorder configurations).

The linear fits in Fig. 5(a) are calculated in various regions of the ME phase, and the localized phase of the model, as summarized in Table II, along with the other three models, which we present in the Appendixes.

The nonmonotonic behavior of $I_{0}$ in Fig. 5(b) points out that the nonmonotonicity in the steady-state imbalance, as seen here and in Ref. [43], is not a finite-size effect, but rather tells us that the imbalance is no longer a trivial function of the number of localized and extended single-particle states.

\section{Dimensionless parameters $\epsilon$ and $\epsilon^{\prime}$}

The anomalies in the steady-state imbalance in Ref. [43] and Fig. 5(b) show that $I_{0}$ is not a trivial function of the fraction of localized states. The degree to which an initial CDW can relax depends on the single-particle states the particles occupy, and the localization length of these states should dictate how far the particles can travel and how much the CDW can relax. A useful quantity is the dimensionless parameter $\epsilon$ defined in Ref. [44], which calculates how strongly the single-particle localized states are localized versus how strongly the single-particle extended states are extended. The definition of this parameter was presented in Sec. III, along with the new parameter $\epsilon^{\prime}$ for calculating the strength/degree of localization of the single-particle localized states. The plots for the two parameters are presented in Fig. 6.

The plot for $\epsilon^{\prime}$ shows that even within the localized phase, not all the states are uniformly localized, and some are localized more than the others, which is expected. But it is the presence of this nonuniformity in the localization length of these states that gives rise to the nonuniformity of the steady-state imbalance in the localized phase. The trend of $I_{0}$ 
TABLE II. Finite-size dependence of steady-state imbalance. The values shown in the third column of the table are of the representative parameters of the corresponding model identified in the second column. The corresponding type of single-particle spectrum is given in the first column. The points marked with “*” are the multiple ME zones (Appendix B). Note that in all cases the steady-state imbalance has $1 / L$ scaling. For the critical cases (i.e., third and fourth row of the table), this $1 / L$ scaling happens even at small system sizes as per our numerics.

\begin{tabular}{lrr}
\hline \hline Type of spectrum & Model & Parameters $(\lambda, \alpha)$ \\
\hline Mobility edge & 1 & $(0.5,0.8),(1.2,0.8)$ \\
& $(0.5,0.8),(0.75,0.8)$ & $(0.8,0.2),(1.4,0.2),{ }^{*}(1.7,0.5)$ \\
& 2 & $(0.75,0.2),(0.9,0.8)$ \\
Localized & 3 & $(1.8,0.4)$ \\
& 4 & $(1.5,0.1),(1.5,0.8)$ \\
& 1 & $(1.7,0.1),(2.0,0.2)$ \\
AAH critical point & 2 & $(1.4,0.3),(1.8,0.2)$ \\
Critical point (ME to localized transition) & 3 & $(1.0,0)$ \\
\hline \hline
\end{tabular}

in Fig. 5 in the localized phase is consistent with that of $\epsilon^{\prime}$. This is also the case for the other three models, presented in the Appendixes.

In the mobility edge phase, there is a competition between the extended and localized states. The extended states allow the particle to move through the entire length of the lattice and hence allow the CDW to relax completely, whereas the localized states restrict the motion of the particles and create a configuration that is closer to the initial condition. Thus, the $\epsilon$ parameter measures how effective this competition is by taking into account not only how many of these extended/localized states are present, but also how much each state contributes to the CDW relaxation.

A direct tallying of Figs. 5(b) and 6 shows that this is indeed the case. In the mobility edge phase, $I_{0}$ follows the trend of the parameter $\epsilon$, increasing in accordance with $\epsilon$ for $\lambda=0.5,1,1.2,1.4$, and decreasing in accordance with $\epsilon$ for $\lambda=1.8$.

One key thing to notice from Fig. 5 is that the behavior of $I_{0}$ is also dependent on the phase the model is in. In other words, it increases or decreases differently in the ME phase and the localized phase. Also, a change in the trend of $I_{0}$ as we increase $\alpha$, keeping $\lambda$ fixed, indicates that there has been a phase transition. This is expected from the imbalance diagrams as well, in Figs. 1-4. A more convincing point for the importance of these two parameters to explain the steady-state imbalance is made by the imbalance phase diagram of model 4. In model 4 (Fig. 4), there is a fingerlike projection of a low imbalance region into the localized phase, and the imbalance is not constant across $\lambda>1$, although every $(\lambda, \alpha)$ for $\lambda>1$ corresponds to a localized phase. Plots of $\epsilon$ and $\epsilon^{\prime}$ in Fig. 7 show how this might occur.

The $\epsilon^{\prime}$ is very low for all $\lambda$ near $\alpha=0$. The eigenstates are not very strongly localized - they have a very large localization length, of order $N$ (system size), and hence the imbalance is close to 0 . The localized character of these states starts getting stronger and stronger, and the parameter $\epsilon^{\prime}$ peaks with this, reaching its maximum in between $\alpha=0.7$ and $\alpha=0.8$.

This is followed by a dip in $\epsilon^{\prime}$ around $\alpha=0.9$, where the states seem to completely lose their localized form, and this is the region of low imbalance that is seen in Fig. 4(b). The states pick up their localization character soon after, and the localization strength reaches its maximum when $\alpha=1$, and the system hits the AAH limit.

Table II contains the summary of the finite-size scaling explored in this paper for different models. At the critical point, we find from numerics that $1 / L$ scaling holds even at small system sizes, and in the localized and mobility edge phase, this is the leading order correction term.

\section{SUMMARY}

To summarize, we have seen that by performing exact diagonalization on systems of length up to 900 sites, we may define a metal-to-insulator transition in noninteracting one-dimensional quasiperiodic systems with the population imbalance as an order parameter. A value close to zero indicates a metallic (ergodic) phase, whereas a value close to the initial starting point defines an insulating (MBL) phase. An intermediate value of the imbalance indicates restrictive relaxation of the charge density wave, and hence the presence of both localized and delocalized states (i.e., a mobility edge phase). The steady-state imbalance has a linear dependence on the inverse of the system size, $1 / L$ to leading order for all the systems studied here, in the localized and mobility edge phase, i.e., wherever the imbalance value was high enough to allow us to determine such a dependence. We see that while this $1 / L$ behavior holds even at small system sizes at the critical point, it is also the leading order correction term for the mobility edge phase and the localized phase for all the systems considered above. We have extracted the thermodynamic behavior of imbalance from this finite-size scaling.

The steady-state imbalance shows nonmonotonic behavior, as found in the phase diagram of imbalance for model 1 in Ref. [43]. We see that although we cross from a region of partial localization of the single-particle spectrum to complete localization, the imbalance drops, around $\lambda=1.2$. Model 2 (see the Appendixes) illustrates this in a better way, where we see nonmonotonic behavior even in the localized phase, where one might naively have expected the imbalance to roughly the stay same, as all the states are localized (Fig. 9). This indicates that the fraction of localized states is not the only factor 

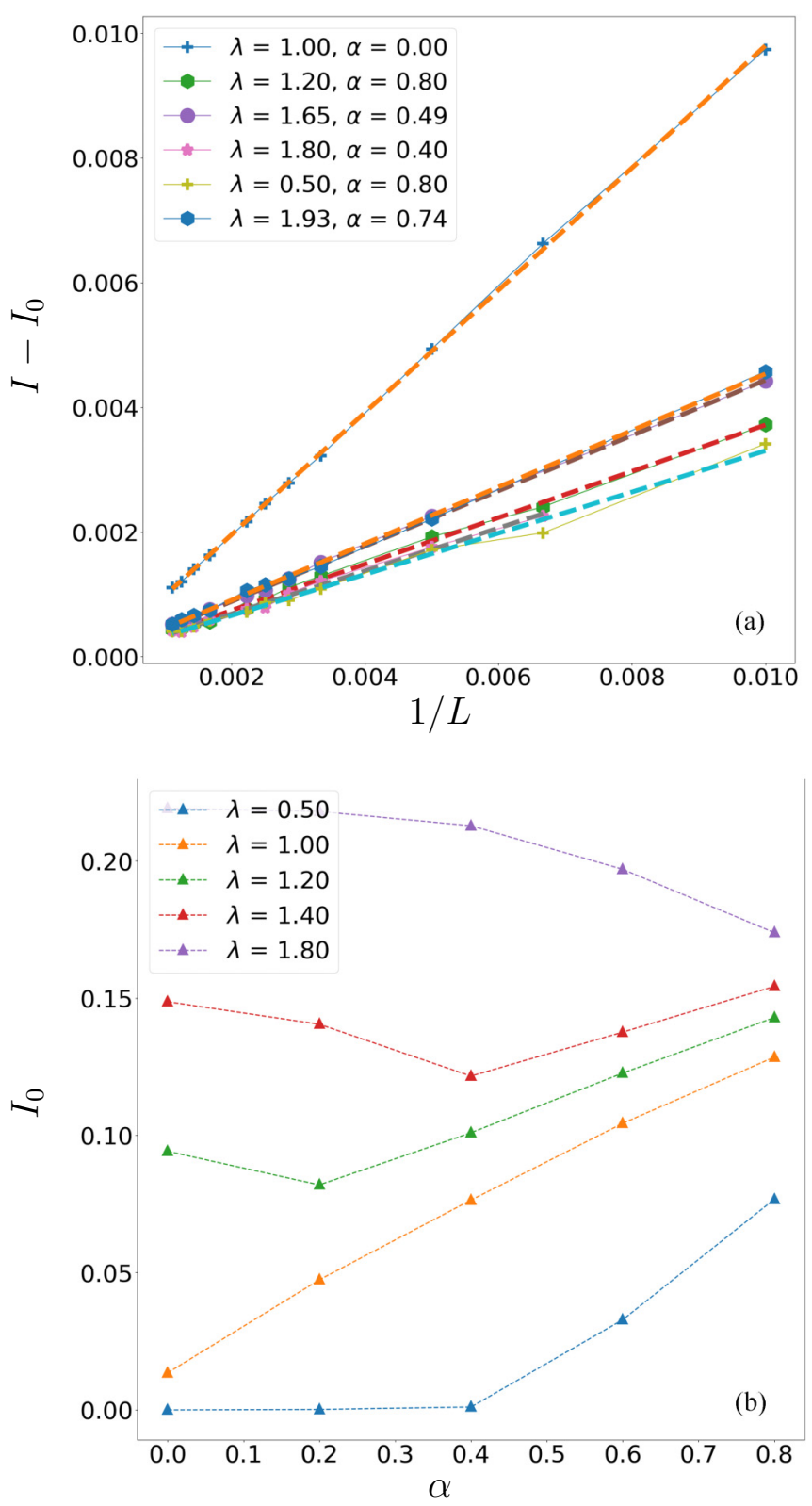

FIG. 5. (a) Steady-state imbalance vs $1 / L$ ( $L$ is the system size) for model 1 (averaged over 100 realizations). The residual value at the limit $L \rightarrow \infty, I_{0}$, has been subtracted to make the plots converge at $L \rightarrow \infty$, which makes the $1 / L$ linear scaling clear. (b) Thermodynamic limit of steady-state imbalance $I_{0}$ for model 1 , averaged over 100 realizations, as a function of $\alpha$ for fixed $\lambda$ 's, obtained from the linear fits for the top panel. Notice that there is a change in trend of $I_{0}$ whenever there is a phase transition from a localized to mobility edge phase, for $\lambda=1.2$ and $\lambda=1.4$.

deciding the relaxation of the CDW, but also how effectively the localized states are localized, as this decides the length over which the particles may move.

We calculate the quantity $\epsilon$ [44], which is a quantifier of how strongly localized the single-particle localized states are versus how strongly delocalized the single-particle delocalized states are. A larger value implies a larger influence of the localized states, and hence a higher value of imbalance. The thermodynamic limit of the imbalance $I_{0}$ follows this
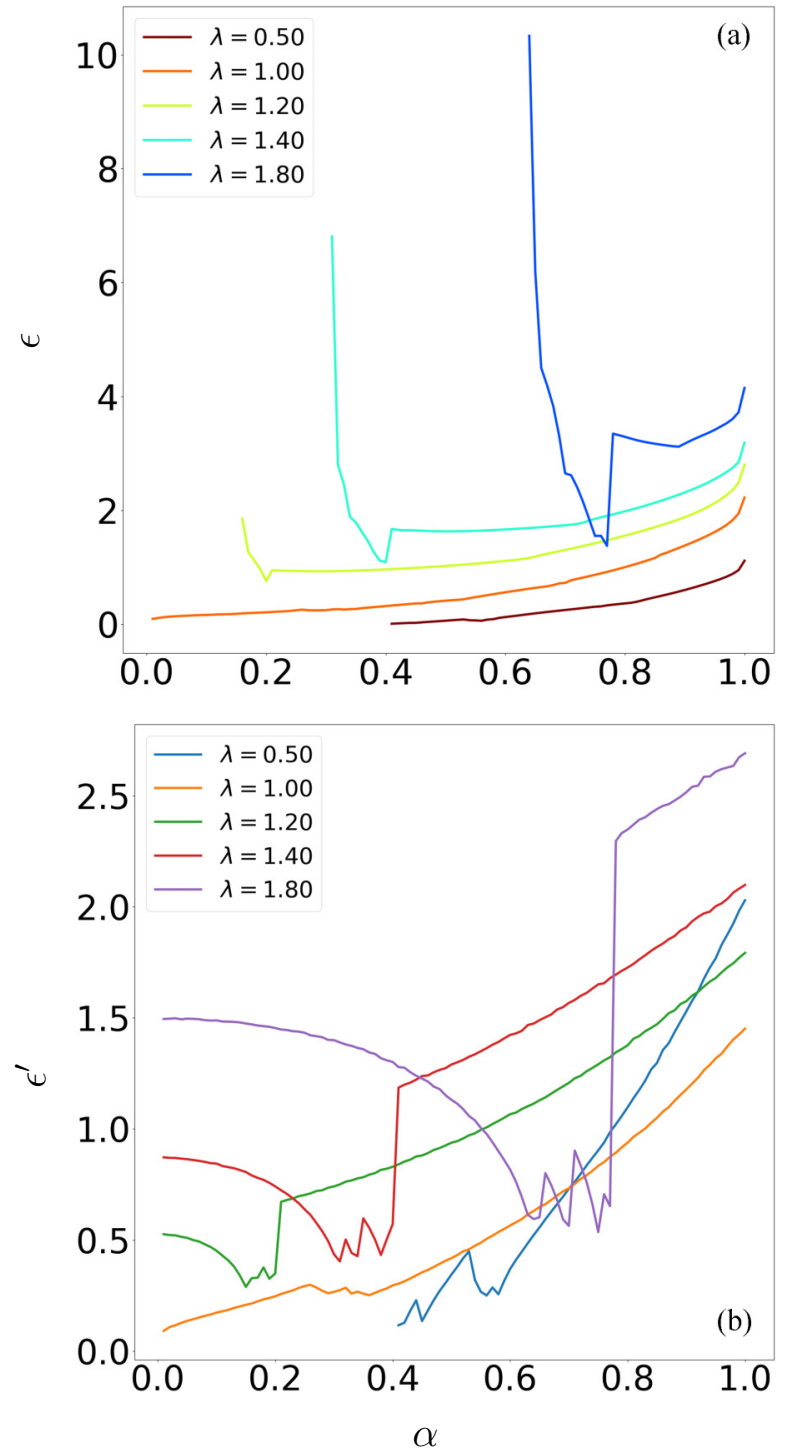

FIG. 6. (a) $\epsilon$ [Eq. (23)] for model 1 , for $L=256$, averaged over 100 realizations. $\epsilon$ is calculated only in the mobility edge phase, along a constant $\lambda$ line in the phase diagram. (b) $\epsilon^{\prime}$ [Eq. (24)] for different values of $\lambda$. In the localized phase, it is calculated over the entire spectrum, whereas in the mobility edge phase it is calculated only over states below the mobility edge that are localized. Note that both $\epsilon$ and $\epsilon^{\prime}$ are 0 in the delocalized phase.

parameter in the mobility edge phase for all the systems we considered, except for model 3 , where the calculation is unfeasible due to an absence of analytical formula for the critical point/mobility edge.

The definition of $\epsilon$ renders it infinity in the localized phase, and hence, if one is interested in the localized phase, a different parameter $\epsilon^{\prime}$ may be used, which tells us how strongly localized the single-particle localized states are. This quantifies the degree of movement of the particles in the localized phase and explains the nonmonotonicity, such as the one in Fig. 9(c) in the localized phase. The imbalance follows this parameter in the localized phase for all the systems we considered, except for model 3, where the calculation is unfeasible. 

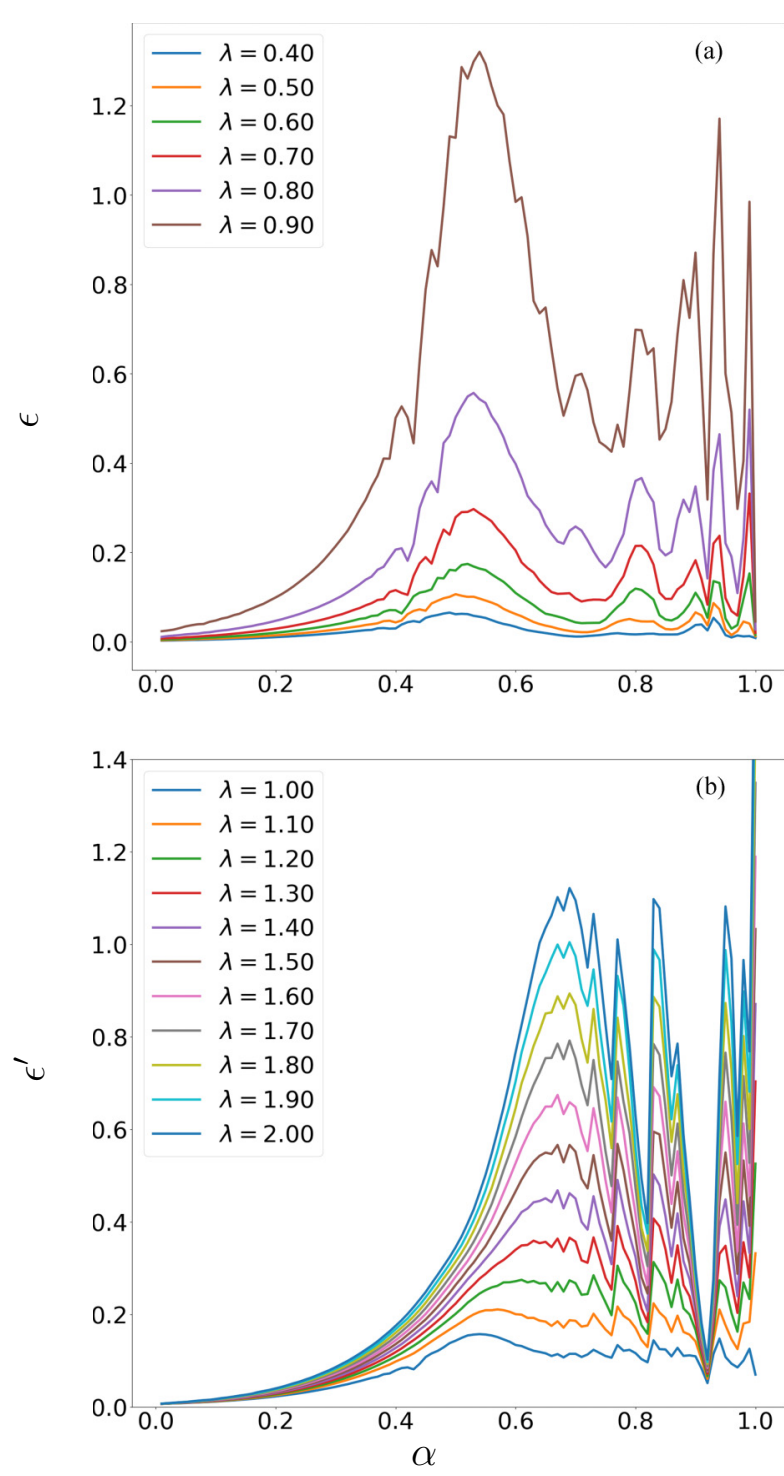

FIG. 7. (a) $\epsilon$ for model 4 , for $L=256$, averaged over 100 realizations. Note the relative peaks in $\epsilon$ around $\alpha=0.5$, in the mobility edge phase, although the imbalance phase diagram is structureless in this region and does not show any region of relatively high imbalance. (b) $\epsilon^{\prime}$ for model 4 averaged over 100 disorder realizations, calculated for different values of $\lambda$ in the localized phase. Note the dip in the value for $\epsilon^{\prime}$ for all $\lambda$ 's around $\alpha=0.9$. This corresponds to a fingerlike projection of the low imbalance region into the localized phase [Fig. 4(b)].

To conclude, the parameters $\epsilon$ and $\epsilon^{\prime}$ play a pivotal role in understanding the phase diagrams of our models.

As a future outlook, extending these studies to the interacting case will be very interesting both from a theoretical and an experimental perspective. A rigorous theoretical understanding of the $1 / L$ behavior of the steady-state imbalance remains an open question. Studying higher spatial dimensional systems [49] through the lens of the diagnostics we introduced is an important future goal.
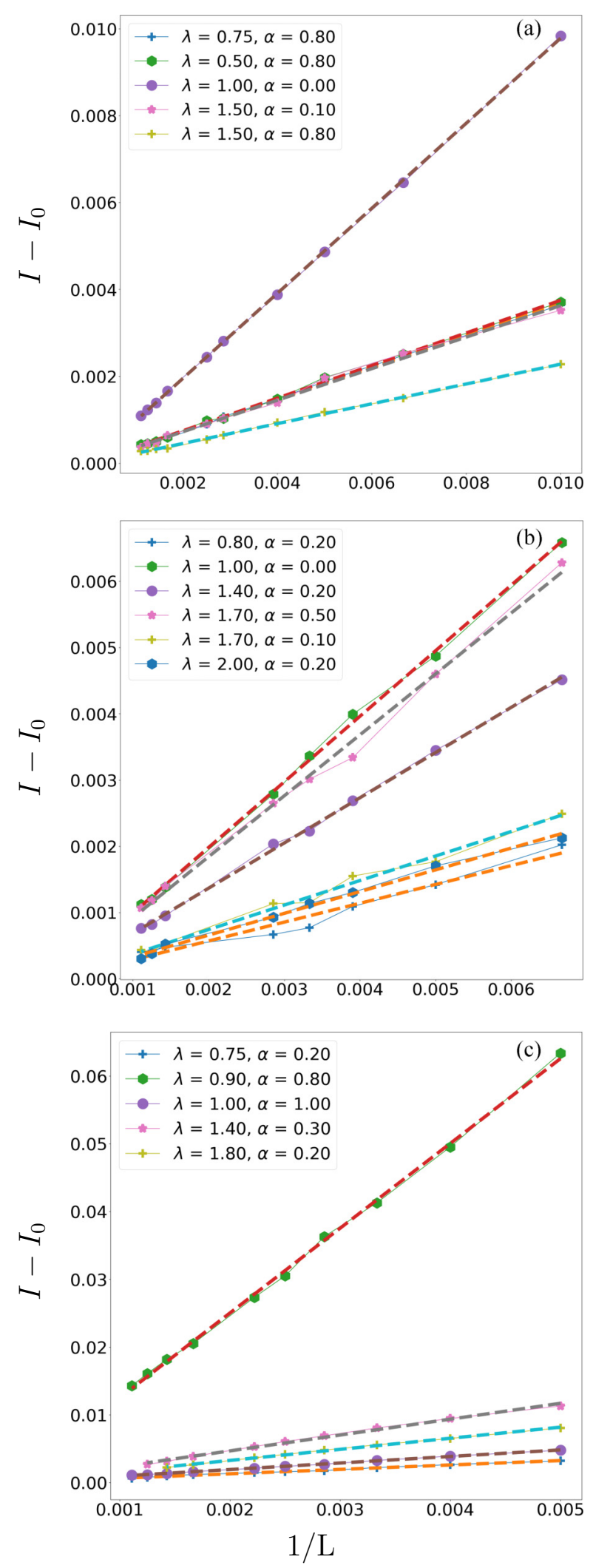

FIG. 8. (a) System size dependence of steady-state imbalance, averaged over 100 disorder realizations for (a) model 2, (b) model 3, and (c) model 4 . The residual value at the thermodynamic limit has been subtracted to make the plots converge at $L \rightarrow \infty$ and to make the $1 / L$ linear scaling clear. 

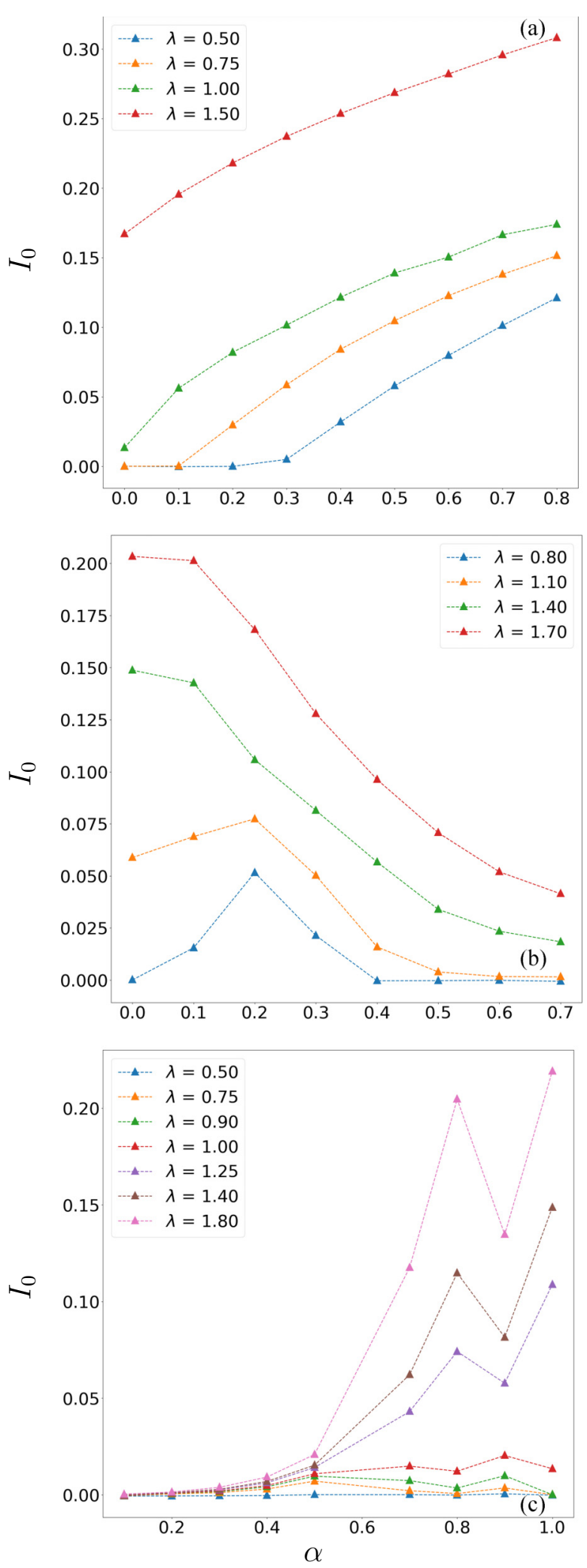

FIG. 9. Thermodynamic limit of steady-state imbalance $I_{0}$ for different $\lambda$ 's with increasing $\alpha$, from similar linear fits (averaged over 100 disorder realizations) up to leading order in $1 / L$ as in Fig. 8. All the linear fits from which we extract $I_{0}$ have not been presented in Fig. 8 for the sake of brevity. Plots are for (a) model 2, (b) model 3, and (c) model 4. The various regions explored in Figs. 8 and 9 can be looked up in Table II and the phase diagrams in Figs. 2-4.

\section{ACKNOWLEDGMENTS}

We would like to thank R. Modak, A. Purkayastha, S. Ghosh, F. Weiner, F. Evers, A. Acharya, and S. Sarangi for useful discussions. M.K. would like to acknowledge support from the Project No. 6004-1 of the Indo-French Centre for the Promotion of Advanced Research (IFCPAR), Ramanujan Fellowship (SB/S2/RJN-114/2016), SERB Early Career Research Award (ECR/2018/002085), and SERB Matrics Grant No. (MTR/2019/001101) from the Science and Engineering Research Board (SERB), Department of Science and Technology (DST), Government of India. S.M. thanks the Quantum Information Science and Technology (QuST) initiative of the DST, Government of India. We are grateful to the ICTS-TIFR high performance computing facility. M.K. acknowledges support of the Department of Atomic Energy, Government of India, under Project No. RTI4001.

\section{APPENDIX A: SYSTEM SIZE SCALING FOR OTHER MODELS}

The system size scaling obtained for model 1 is quite robust and holds generally for the other models we consider in this paper (Table I). Figure 9(a) shows that even for model 2 in the strict localized phase, the imbalance does change from one point to the other, although the number of localized states stays the same. This further supports the hypothesis that the number of single-particle localized states is not the only deciding factor for the steady-state value of imbalance, but also how effectively they are localized against the delocalized states (refer to Secs. IV B and IV C for the anomalies in model 1 and the subsequent justification). The steady-state value of imbalance suggests that the localized states close to $\lambda=1$ are weakly localized, and they get more and more localized as the system moves deeper into the localized phase.

Figure 9(b) tells a similar story to the phase diagram (Fig. 3) for model 3, in that there is a tiny region of high imbalance around $\alpha=0.2$ for $\lambda<1$. For $\lambda>1$, the imbalance decreases from the localized phase to the mobility edge phase.

While the AAH critical point has $1 / L$ scaling even for small system sizes, for the three remaining models (refer to Table II), the localized and mobility edge phases also show a leading order $1 / L$ scaling (Fig. 8). For model 4 , the value of imbalance is fairly low even in the localized phase for all system sizes, and hence the thermodynamic limit of imbalance asymptotes to a value close to zero, except for a tiny sliver of high imbalance close to $\alpha=0.8$ (Fig. 4).

While model 1 showed a nonmonotonicity of imbalance in the localized phase and mobility edge, this is not unique to model 1, and can be seen in the other models as well. Calculation of $I_{0}$ from the linear fits in Fig. 8 shows this nonmonotonicity. The phase diagram of model 4, Fig. 4(a), shows a uniform localized phase for all values of $\alpha$ for $\lambda>1$. However, the steady-state imbalance showed that the nature of localized states is drastically different. They are very weakly localizing near the Bloch limit $\alpha=0$, where they still retain some of their plane-wave nature. This gives rise to a very low value of imbalance, and although localized, this model exhibits a value that is close to 0 even in the thermodynamic limit. The imbalance, however, spikes up close to $\alpha=0.8$, 

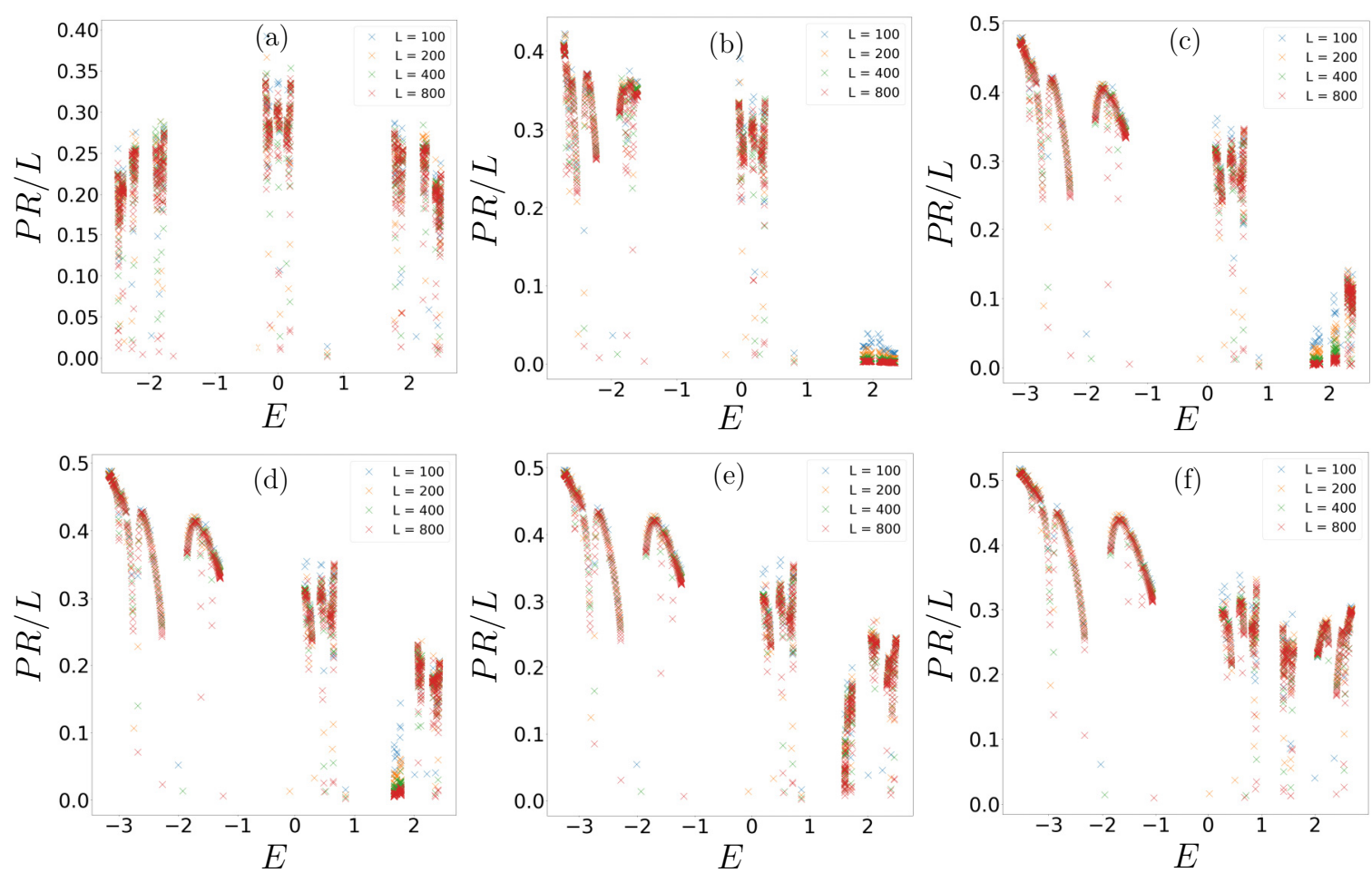

FIG. 10. PR of single-particle states, defined in Eq. (15) for model 3, scaled by system size. We recollect that PR is $O(1)$ for localized states and $O(L)$ for delocalized states [see Eq. (21)]. All the plots are for $\lambda=0.9$, showing a transition from a delocalized to mobility edge to a delocalized phase again through appearances and the merger of multiple mobility edges. (a) $\alpha=0$ : The AAH limit, where all states are delocalized. (b) $\alpha=0.15$ : A single mobility edge appears separating the $O(L)$ extended states from $O(1)$ localized states. For (c) $\alpha=0.35$ and (d) $\alpha=0.4$, two mobility edges appear separating a region of localized states in the spectrum around $E=2$ from extended states on either sides. In other words, around $E=2$ there is a region of localized states. (e) $\alpha=0.45$ : The mobility edges merge to give a special band of states near $E=1.6$. (f) $\alpha=0.6$ : The mobility edges have merged, giving a delocalized phase again.

before dipping at $\alpha=0.9$ before the model goes to the AAH limit. The nature for this sudden drop is not very clear yet.

\section{APPENDIX B: PARTICIPATION RATIOS FOR MODEL 3}

In Sec. IV A, it was mentioned that for model 3, the delocalization to mobility edge transition, and the transition back to the delocalized phase, was due to the appearance and merging of multiple mobility edges. These transitions may be seen from following any vertical cut in the region $0.75<\lambda<1.0$ in Fig. 3(b). The participation ratio, defined in Eq. (15), describes the appearance of mobility edges as an approximate energy value that separates the states with extensive and intensive scaling with system size, i.e., it is $O(L)$ for delocalized states, and $O(1)$ for localized states. For various parameters this intricate transition is observed for model 3, in the phase $\lambda<1$. In Fig. 10, one such transition is shown for $\lambda=0.9$. As we increase $\alpha$, the system moves from a delocalized phase to a mobility edge phase. At higher $\alpha$, a second mobility edge appears, and as $\alpha$ is increased further, these two edges merge together to transition the system back into the delocalized phase.
[1] P. W. Anderson, Phys. Rev. 109, 1492 (1958).

[2] A. Lagendijk, B. Tiggelen, and D. Wiersma, Phys. Today 62 (8), 24 (2009).

[3] A. Aspect and M. Inguscio, Phys. Today 62 (8), 30 (2009).

[4] G. Semeghini, M. Landini, P. Castilho, S. Roy, G. Spagnolli, A. Trenkwalder, M. Fattori, M. Inguscio, and G. Modugno, Nat. Phys. 11, 554 (2015).

[5] S. Aubry and G. André, Ann. Israel Phys. Soc. 3, 18 (1980).

[6] P. G. Harper, Proc. Phys. Soc., London, Sect. A 68, 879 (1955).

[7] D. R. Hofstadter, Phys. Rev. B 14, 2239 (1976).

[8] S. Ostlund, R. Pandit, D. Rand, H. J. Schellnhuber, and E. D. Siggia, Phys. Rev. Lett. 50, 1873 (1983).
[9] A. Purkayastha, S. Sanyal, A. Dhar, and M. Kulkarni, Phys. Rev. B 97, 174206 (2018).

[10] M. Schreiber, S. S. Hodgman, P. Bordia, H. P. Lüschen, M. H. Fischer, R. Vosk, E. Altman, U. Schneider, and I. Bloch, Science 349, 842 (2015).

[11] H. P. Lüschen, P. Bordia, S. Scherg, F. Alet, E. Altman, U. Schneider, and I. Bloch, Phys. Rev. Lett. 119, 260401 (2017).

[12] H. P. Lüschen, P. Bordia, S. S. Hodgman, M. Schreiber, S. Sarkar, A. J. Daley, M. H. Fischer, E. Altman, I. Bloch, and U. Schneider, Phys. Rev. X 7, 011034 (2017).

[13] J. Major, G. Morigi, and J. Zakrzewski, Phys. Rev. A 98, 053633 (2018). 
[14] E. G. Guan, H. Yu, and G. Wang, Phys. Lett. A 384, 126152 (2020).

[15] Y. E. Kraus and O. Zilberberg, Phys. Rev. Lett. 109, 116404 (2012).

[16] Y. E. Kraus, Y. Lahini, Z. Ringel, M. Verbin, and O. Zilberberg, Phys. Rev. Lett. 109, 106402 (2012).

[17] A. Sinha, M. M. Rams, and J. Dziarmaga, Phys. Rev. B 99, 094203 (2019).

[18] H. P. Lüschen, S. Scherg, T. Kohlert, M. Schreiber, P. Bordia, X. Li, S. Das Sarma, and I. Bloch, Phys. Rev. Lett. 120, 160404 (2018).

[19] T. Kohlert, S. Scherg, X. Li, H. P. Lüschen, S. Das Sarma, I. Bloch, and M. Aidelsburger, Phys. Rev. Lett. 122, 170403 (2019).

[20] F. A. An, K. Padavić, E. J. Meier, S. Hegde, S. Ganeshan, J. Pixley, S. Vishveshwara, and B. Gadway, Phys. Rev. Lett. 126, 040603 (2021).

[21] R. Wang, X. M. Yang, and Z. Song, arXiv:1912.04506.

[22] Y. Wang, X. Xia, L. Zhang, H. Yao, S. Chen, J. You, Q. Zhou, and X.-J. Liu, Phys. Rev. Lett. 125, 196604 (2020).

[23] X. Li, S. Ganeshan, J. H. Pixley, and S. Das Sarma, Phys. Rev. Lett. 115, 186601 (2015).

[24] R. Modak and S. Mukerjee, Phys. Rev. Lett. 115, 230401 (2015).

[25] S. Ganeshan, J. H. Pixley, and S. Das Sarma, Phys. Rev. Lett. 114, 146601 (2015).

[26] M. Sun, G. Wang, N. Li, and T. Nakayama, Europhys. Lett. 110, 57003 (2015).

[27] M. Johansson, Europhys. Lett. 112, 17002 (2015).

[28] D.-L. Deng, S. Ganeshan, X. Li, R. Modak, S. Mukerjee, and J. Pixley, Ann. Phys. 529, 1600399 (2017).

[29] S. Nag and A. Garg, Phys. Rev. B 96, 060203(R) (2017).

[30] J. Biddle, B. Wang, D. J. Priour, Jr., and S. Das Sarma, Phys. Rev. A 80, 021603(R) (2009).
[31] J. Biddle and S. Das Sarma, Phys. Rev. Lett. 104, 070601 (2010).

[32] X. Li, X. Li, and S. Das Sarma, Phys. Rev. B 96, 085119 (2017).

[33] X. Li, J. H. Pixley, D.-L. Deng, S. Ganeshan, and S. Das Sarma, Phys. Rev. B 93, 184204 (2016).

[34] S. Gopalakrishnan, Phys. Rev. B 96, 054202 (2017).

[35] H. Yao, H. Khoudli, L. Bresque, and L. Sanchez-Palencia, Phys. Rev. Lett. 123, 070405 (2019).

[36] H. Yao, T. Giamarchi, and L. Sanchez-Palencia, Phys. Rev. Lett. 125, 060401 (2020).

[37] S. Lellouch and L. Sanchez-Palencia, Phys. Rev. A 90, 061602(R) (2014)

[38] A. Szabó and U. Schneider, Phys. Rev. B 101, 014205 (2020).

[39] A. De Luca, B. L. Altshuler, V. E. Kravtsov, and A. Scardicchio, Phys. Rev. Lett. 113, 046806 (2014).

[40] Y. Baum, E. van Nieuwenburg, and G. Refael, APS March Meeting Abstracts, APS Meeting Abstracts, Vol. 2019 (2019), p. R25.003.

[41] D. Basko, L. Aleiner, and B. Altshuler, in Problems of Condensed Matter Physics, edited by A. L. Ivanov and S. G. Tikhodeev (Oxford University Press, Oxford, U.K., 2006), p. 50 .

[42] S. Das Sarma, S. He, and X. C. Xie, Phys. Rev. B 41, 5544 (1990).

[43] A. Purkayastha, A. Dhar, and M. Kulkarni, Phys. Rev. B 96, 180204(R) (2017).

[44] R. Modak, S. Ghosh, and S. Mukerjee, Phys. Rev. B 97, 104204 (2018).

[45] C. M. Soukoulis and E. N. Economou, Phys. Rev. Lett. 48, 1043 (1982).

[46] J. Biddle, D. J. Priour, B. Wang, and S. Das Sarma, Phys. Rev. B 83, 075105 (2011).

[47] M. Berry and J. Goldberg, Nonlinearity 1, 1 (1988).

[48] D. J. Thouless, Phys. Rev. Lett. 61, 2141 (1988).

[49] T. Devakul and D. A. Huse, Phys. Rev. B 96, 214201 (2017). 